\title{
Estudo da série histórica de mortalidade por suicídio no Espírito Santo (de 1980 a 2006)
}

\author{
A study of the historic series on mortality per suicide \\ in Espírito Santo, Brazil (from 1980 to 2006) \\ Luciene Bolzam Macente', Eliana Zandonade’
}

\section{RESUMO}

Objetivo: Estudar a série histórica de suicídio no Estado do Espírito Santo (1980 a 2006) e suas estratificações. Métodos: Estudo descritivo, retrospectivo, de série histórica. Foram incluídos os óbitos registrados no Sistema de Informação sobre Mortalidade como suicídio, de residentes no Espírito Santo (1980 a 2006). Foram construídas as séries históricas e calculados os coeficientes de mortalidade por suicídio para o período. Procedeu-se à padronização das taxas de mortalidade pelo método direto, em que a população do censo IBGE-2000 foi considerada padrão. Para cálculo das taxas, utilizou-se o Programa Excel 7.0. As equações de tendência linear e as estatísticas de ajuste de modelo (valor de $R^{2}$ e o valor $p$ do teste $F$ de adequação do modelo) foram obtidas do programa Statistical Package for Social. Sciences. O nível de significância adotado foi de 5\%. Resultados: A tendência de crescimento das

\section{Palavras-chave}

Suicídio, suicídio/ tendências, sistemas de informação, epidemiologia. taxas de suicídio no Estado foi de 24,9\%. Para os homens, essa tendência de crescimento foi de 23,8\%. As faixas etárias com tendência de crescimento foram aquelas entre 10 e 14 anos e acima de 30 anos até 59 anos. Conclusão: $\bigcirc$ suicídio foi mais frequente entre os homens, e as principais faixas etárias com tendência de crescimento foram aquelas entre 30 e 59 anos.

\begin{abstract}
Objective: Study historical series of suicide in Espirito Santo (1980 to 2006) and its stratifications. Methods: A descriptive, retrospective study based on historical series of events. The registered deaths were included from the System of Information on Mortality per Suicide by residents of Espírito Santo (1980 to 2006). The historical series were put together and the mortality coefficients per suicide were calculated for a certain period. Afterwards, the direct method was used in order to fit death table numbers into a pattern - in this stage, the population number was taken from data published by the Brazilian Institute of Geography and Statistics in 2000. So the death tables could be calculated, the Microsoft Excel software was used. The equations of linear tendencies and the statics of adjustment into the model ( $R^{2}$ value and $p$-value of model adequacy) were collected from the Statistical Package for Social. Sciences software. The level of significance adopted was of 5\%. Results: The tendency of growth of a death table per suicide in Espirito Santo was 24.9\%. Regarding men, 23.8\%. The age group between 10 and 14 years and those above 30 until 59 years old had a strong growth tendency. Conclusion: Suicidal acts were more common among men. The main age group which may be likely to have a growth in is the one ranging from 30 and 57 years old.
\end{abstract}

$$
\begin{gathered}
\text { Recebido em } \\
\text { 14/12/2010 } \\
\text { Aprovado em } \\
\text { 27/6/2011 }
\end{gathered}
$$

\section{Keywords}

information systems, epidemiology.
1 Universidade Federal do Espírito Santo (Ufes), Programa de Pós-graduação em Saúde Coletiva.

Endereço para correspondência: Luciene Bolzam Macente

Rua Luiz Disepi, 133 - Vista da Serra I - 29176-420 - Serra, ES

Tels.: (27) 3251-2752/(27) 9973-5958

E-mail: lucienebolzam@hotmail.com 


\section{INTRODUÇÃO}

Dados epidemiológicos mundiais indicam que os comportamentos suicidas constituem um importante problema de saúde pública. Segundo previsão da Organização Mundial da Saúde (OMS), em 2020, aproximadamente 1,53 milhão de pessoas no mundo morrerão por suicídio e um número de 10 a 20 vezes maior de pessoas tentará suicídio. Isso representa 1 morte por suicídio a cada 20 segundos e 1 tentativa de suicídio a cada 1 a 2 segundos'

Dados da OMS indicam ainda que as mortes por suicídio aumentaram 60\% nos últimos 45 anos $^{2}$. O suicídio está entre as dez principais causas de óbito para todas as pessoas maiores de 5 anos de idade. Em todos os países onde há informações fidedignas sobre a mortalidade, esse evento se encontra entre as 3 principais causas de morte para as pessoas de ambos os sexos com idade entre 15 e 34 anos $^{3}$.

No Brasil, as taxas de suicídio entre 1980 e 2000 foram consideravelmente mais baixas do que as de muitos países. Destaca-se que, quando se consideram as taxas de suicídio (número de casos de suicídio/100.000 habitantes), o Brasil ocupa o $71^{\circ}$ lugar na classificação mundial, mas, em números absolutos de mortes por suicídio, o Brasil ocupa a 9a posição no ranking mundial ${ }^{4}$.

A descrição do perfil epidemiológico do suicídio no Brasil vem crescendo significativamente nas últimas décadas. Estudo realizado por Gawryszewski et al. aponta para o fato de que, no ano 2000, o suicídio foi responsável por 6.778 óbitos, o que correspondeu a $5,7 \%$ do total de mortes, com taxa de mortalidade igual a 4,0/100 mil habitantes. Vale salientar que, entre os homens, a taxa de mortalidade foi de 5,0 e, entre as mulheres, de 1,6/100 mil habitantes ${ }^{5}$. Em 2005, Mello-Santos et al. também publicaram estudo sobre as taxas nacionais de morte por suicídio entre os anos de 1980 e 2000 . Os autores encontraram uma média de 3 a 4 suicídios/100.000 habitantes no Brasil, sendo a incidência 4 vezes maior entre homens e com taxas crescentes nas faixas etárias mais jovens ${ }^{6}$. Souza et al. ${ }^{7}$ encontraram, em pesquisa epidemiológica em diversas capitais do Brasil, o suicídio como sexta causa de morte em populações jovens e urbanas (15 a 24 anos).

No que se refere à distribuição dos óbitos por suicídio no Brasil, verifica-se que os coeficientes variam muito entre as regiões do país, pois, enquanto a Região Sudeste concentra 50\% dos registros de suicídio, a Região Sul possui os maiores coeficientes de suicídio e a Região Norte os menores índices, não sendo, contudo, possível afirmar precisamente qual a relação entre esses dados e os problemas relacionados à qualidade do registro das informações ${ }^{8,9}$.

Entre os Estados brasileiros com maiores taxas de suicídio, estão o Rio Grande do Sul, Santa Catarina, Paraná, São Paulo e Goiás, em ordem decrescente ${ }^{10,11}$. Na Região Sudeste, segundo dados do Ministério da Saúde para o ano de 2004, o Estado de São Paulo apresentou crescimento das taxas de suicídio entre 1988 a 1994 e queda acentuada no período de 1995 a 2004, ao passo que o Estado com maior crescimento da taxa na região foi o Espírito Santo, com aumento de 50\% no valor da taxa no período de 1980-2004 ${ }^{12}$.

Apesar desses dados, são poucos os estudos que abordam a questão do suicídio no Estado do Espírito Santo. Com exceção de dados do Ministério da Saúde e da United Nations Educational, Scientific and Cultural Organization (Unesco), verifica-se que a discussão sobre o tema gira em torno da mortalidade violenta ou mortalidade por causas externas. Por isso, o presente estudo foi elaborado a partir da necessidade de se desenvolverem estudos mais minuciosos (especialmente em relação a estratificações como sexo, faixa etária, macro e microrregiões) do padrão de mortalidade por suicídio no Estado do Espírito Santo e, com isso, poder comparar os resultados encontrados a estudos já realizados em algumas cidades brasileiras.

Desse modo, o presente estudo possui como objetivo estudar a série histórica de suicídio no Estado do Espírito Santo, de 1980 a 2006, com base em dados secundários do Sistema de Informação sobre Mortalidade (SIM), em relação às seguintes estratificações: sexo, faixa etária, macro e microrregiões.

\section{MÉTODOS}

Todo o processo de pesquisa foi conduzido conforme a Resolução n. 196/96, do Conselho Nacional de Saúde (CNS), tendo sido o projeto aprovado pelo Comitê de Ética e Pesquisa da Universidade Federal do Espírito Santo (Ufes), sob o n. $159 / 09$.

Realizou-se estudo descritivo retrospectivo baseado em dados secundários. Foram incluídos os óbitos de residentes no Estado do Espírito Santo (ES), registrados no SIM, os quais possuíam como causa básica o suicídio, dados esses obtidos no endereço eletrônico do Departamento de Informática do Sistema Único de Saúde (DATASUS) do Ministério da Saúde.

O Estado do Espírito Santo situa-se na Região Sudeste do Brasil, possui $46.077,5 \mathrm{~km}^{2}$ com população estimada para 2009 de 3.487.094 habitantes $^{13}$, distribuídos em 78 municípios. Para fins administrativos, a Secretaria Estadual de Saúde, por meio da lei estadual n. ${ }^{\circ}$ 5120, de 1. ${ }^{\circ}$ de dezembro de 1995, agrega os municípios em 4 Macrorregiões de Planejamento e 12 Microrregiões Administrativas de Gestão.

Os 78 municípios foram divididos e analisados conforme as 4 macrorregiões:

1. Metropolitana;

2. Norte;

3. Noroeste;

4. Sul.

Depois, segundo as 12 microrregiões de saúde:

1. Metropolitana;

2. Polo Linhares; 
3. Metrópole Expandida Sul;

4. Sudoeste Serrana;

5. Central Serrana;

6. Litoral Norte;

7. Extremo Norte;

8. Polo Colatina;

9. Noroeste l;

10. Noroeste II;

11. Polo Cachoeiro:

12. Caparaó.

Para o cálculo do número de suicídios entre os anos de 1980 e 1995, utilizou-se a nona revisão da Classificação Internacional de Doenças (CID-9), que compreende as categorias E950 a E959; para o período entre 1996 e 2006, foi utilizada a décima revisão da Classificação Internacional de Doenças (CID-10), que inclui as categorias X60 a X84 (lesões autoprovocadas intencionalmente).

Para o cálculo do coeficiente de mortalidade por suicídio para o período selecionado, utilizou-se a seguinte fórmula: (número de óbitos por suicídio/população) x 100.000.

O método direto de padronização foi utilizado com o objetivo de controlar o efeito de diferentes estruturas etárias sobre os valores dos coeficientes de mortalidade geral brutos $^{14}$. Para a padronização das taxas de mortalidade pelo método direto, adotou-se a população do censo do Instituto Brasileiro de Geografia e Estatística (IBGE) de 2000 como a padrão para o Estado. Considerou-se necessária a padronização para dispor de taxas de mortalidade que fossem comparáveis entre si e ao longo do período estudado.

Foram construídas séries históricas para o período de 1980 a 2006, sendo esse período selecionado devido à disponibilidade dos dados de mortalidade e população no DATASUS e no IBGE.
As faixas etárias foram descritas conforme apresentação no banco de dados do DATASUS, distribuídas em dez grupos: a) 5 a 9; b) 10 a 14; c) 15 a 19; d) 20 a 29; e) 30 a 39; f) 40 a 49; g) 50 a 59; h) 60 a 69; i) 70 a 79; j) 80 e mais. Em face dos objetivos desta pesquisa, foram excluídas da análise as categorias: menor de 1 ano e 1 a 4 anos.

As taxas de mortalidade foram calculadas utilizando o Programa Excel 7.0. As equações de tendência linear e as estatísticas de ajuste de modelo (valor de $\mathrm{R}^{2}$ e o valor p do teste F de adequação do modelo) foram obtidas do programa Statistical Package for Social. Sciences (SPSS), versão 15.0. O nível de significância adotado foi de 5\%.

\section{RESULTADOS}

No período de estudo (1980 a 2006), ocorreram 2.604 óbitos por suicídio no Estado do Espírito Santo. A incidência de suicídios nesse período variou de 3,5 (ano 1984) a 7,3 (ano 2005) por 100.000 habitantes. Destes, 77,7\% eram do sexo masculino.

Verificou-se, no período estudado, um crescimento geral de $24,9 \%$ ( $p=0,001)$ das taxas de suicídio, tendo sido a taxa de crescimento entre os homens de $23,8 \%$, enquanto, entre as mulheres, a taxa se manteve estável. É o que mostra a Figura 1. Destaca-se que a relação da taxa global média entre homens e mulheres foi de 3,7:1 no período.

No que se refere à faixa etária, verifica-se que 78,1\% dos óbitos por suicídio eram de pessoas com idade entre 20 a 59 anos, destacando-se que, entre as mulheres, 72,3\% possuíam idade entre 15 e 49 anos. É o que demonstra a Figura 2.

Foram realizados modelos de regressão linear simples para os dados da taxa de mortalidade ajustada por faixa etária $(Y)$ segundo a variável ano $(X)$. Foi realizada a padro-

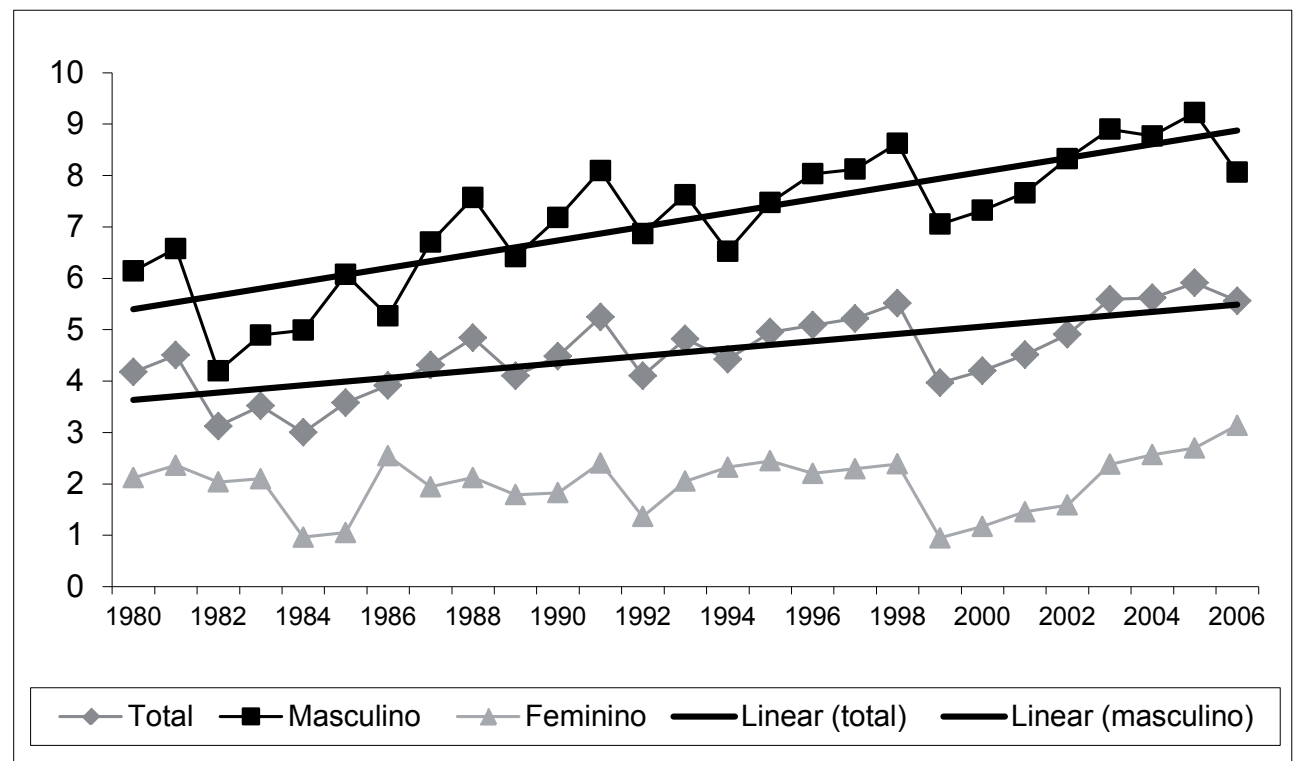

Figura 1. Taxa de suicídio referente ao período de 1980 a 2006 (total) e segundo o sexo (masculino e feminino), com padronização pelo método direto e respectivas linhas de tendência estatisticamente significantes (total e sexo masculino). 
nização pelo método direto para o coeficiente de incidência total e por sexo. As equações dos modelos encontrados, valor de $R^{2}$ e respectivos valores $p$ do teste $F$ indicam, como mostra a tabela 1, que as tendências para algumas variáveis são crescentes e estatisticamente significantes. Para os resultados estatisticamente significantes, calculou-se o percentual de crescimento.

A padronização das populações por grupo etário, pelo método direto, mostrou resultado diferente dos dados não padronizados, sugerindo que as alterações nos indicadores não eram independentes da mudança da estrutura etária da população.

Verificaram-se, ainda, tendências de crescimento das taxas entre as faixas etárias de 30 a 39 anos ( $p=0,019)$; de 40 a 49 anos $(p=0,001)$ e de 50 a 59 anos $(p=0,004)$, com cres- cimento de 16,4, 44,7 e 72,3\%, respectivamente, como demonstra a Figura 3. Para a faixa de 10 a 14 anos, encontramos também uma tendência crescente $(p=0,003)$, passando de uma taxa de 0, em 1980, para 1,4 por 100.000 habitantes, no ano de 2006.

Considerando a distribuição dos casos em termos de macro e microrregiões do Estado, verificou-se tendência de crescimento das taxas nas macrorregiões Metropolitana, Noroeste e Sul, enquanto as taxas da macrorregião Norte se mantiveram estáveis. No que se refere às microrregiões, verifica-se que metade delas (Metropolitana; Sudoeste Serrana; Polo Colatina; Noroeste 1; Noroeste 2; Polo Cachoeiro) apresentaram tendência de crescimento, destacando-se que todas as microrregiões com tendência crescente se localizam nas macrorregiões com tendência crescente.

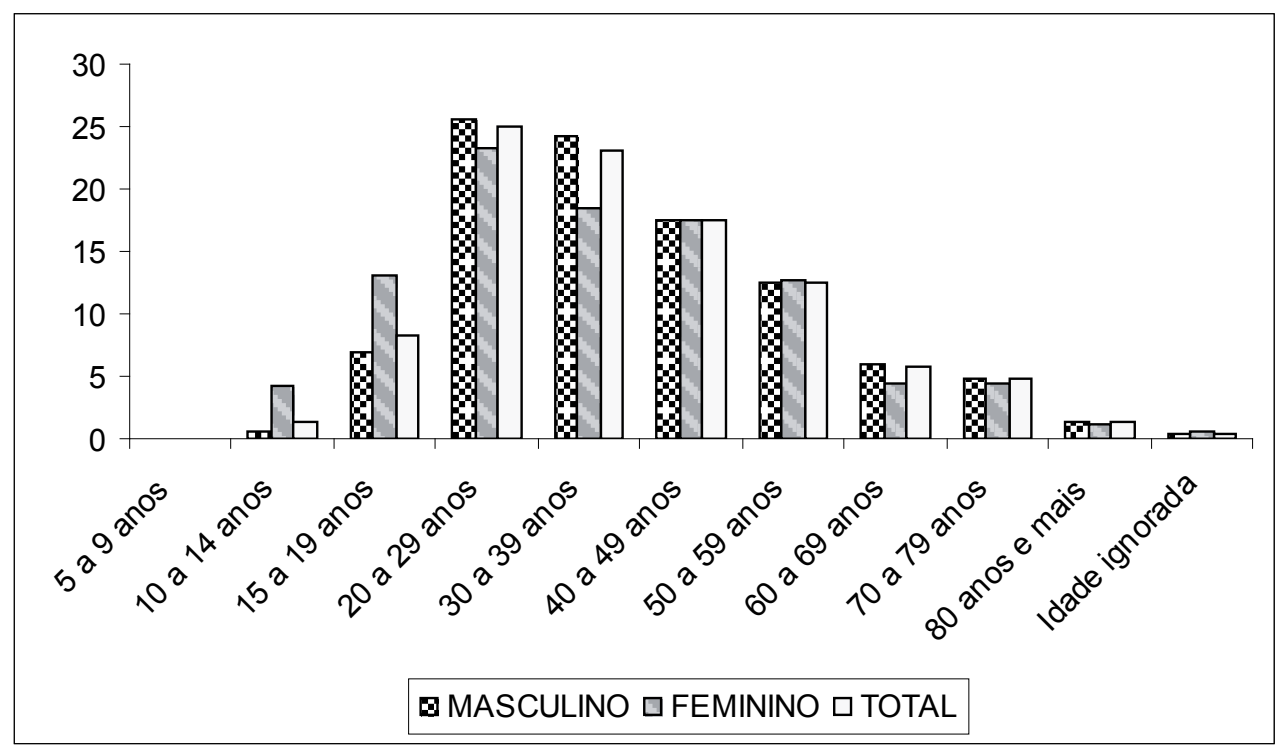

Figura 2. Distribuição do percentual de suicídios por faixa etária e sexo no período de 1980 a 2006.

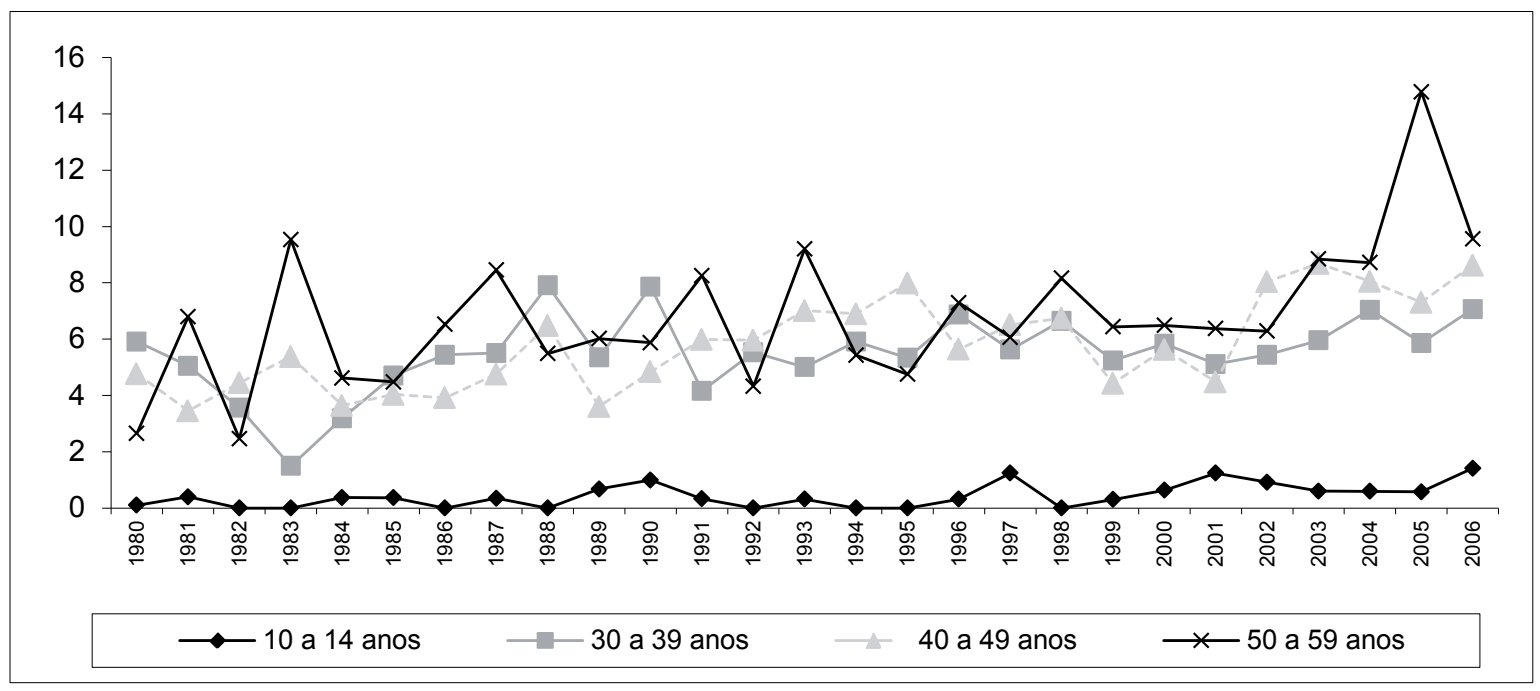

Figura 3. Taxa de suicídio segundo faixas etárias com maior tendência ( 10 a 14 anos, 30 a 39 anos, 40 a 49 anos e 50 a 59 anos) no período de 1980 a 2006. 
Tabela 1. Resultado da análise de tendências e modelo ajustado da taxa de suicídio total, por sexo, faixa etária, macro e microrregião do Estado do Espírito Santo, no período de 1980 a 2006

\begin{tabular}{|c|c|c|c|c|c|}
\hline Variável & Categoria & Modelo & $\mathrm{R}^{2}$ & Valorp & Crescimento (\%) \\
\hline Total $^{*}$ & - & $y=3,561+0,071 x$ & 0,535 & 0,001 & 24,9 \\
\hline \multirow[t]{2}{*}{ Sexo* } & Masculino & $y=5,2612+0,1339 x$ & 0,675 & 0,001 & 23,8 \\
\hline & Feminino & $y=1,7983+0,0151$ & 0,046 & 0,284 & - \\
\hline \multirow[t]{10}{*}{ Faixa etária } & $5 \times 9$ & ** & - & - & - \\
\hline & 10 a 14 & $y=0,014+0,030 x$ & 0,305 & 0,003 & 92,0 \\
\hline & 15 a 19 & $y=2,550+0,013 x$ & 0,008 & 0,659 & - \\
\hline & 20 a 29 & $y=4,242+0,045 x$ & 0,107 & 0,096 & - \\
\hline & 30 a 39 & $y=4,419+0,077 x$ & 0,202 & 0,019 & 16,4 \\
\hline & 40 a 49 & $y=3,753+0,148 x$ & 0,525 & 0,001 & 44,7 \\
\hline & 50 a 59 & $y=4,440+0,169 x$ & 0,289 & 0,004 & 72,3 \\
\hline & 60 a 69 & $y=4,691+0,008 x$ & 0,001 & 0,891 & - \\
\hline & 70 a 79 & $y=5,802+0,150 x$ & 0,086 & 0,139 & - \\
\hline & 80 e mais & $y=5,852-0,002 x$ & 0,000 & 0,990 & - \\
\hline \multirow[t]{4}{*}{ Macro } & Metropolitana & $y=2,427+0,073 x$ & 0,571 & 0,001 & 32,4 \\
\hline & Norte & $y=1,741+0,042 x$ & 0,099 & 0,111 & - \\
\hline & Noroeste & $y=1,618+0,135 x$ & 0,603 & 0,001 & 32,3 \\
\hline & Sul & $y=2,693+0,084 x$ & 0,304 & 0,003 & 57,6 \\
\hline \multirow[t]{12}{*}{ Micro } & Metropolitana & $y=1,522+0,098 x$ & 0,592 & 0,001 & 39,0 \\
\hline & Polo Linhares & $y=2,443+0,025 x$ & 0,033 & 0,364 & - \\
\hline & Sul & $y=2,322-0,009 x$ & 0,002 & 0,820 & - \\
\hline & Sudoeste Serrana & $y=4,453+0,158 x$ & 0,304 & 0,003 & 8,5 \\
\hline & Central Serrana & $y=9,635-0,020 x$ & 0,002 & 0,818 & - \\
\hline & Litoral Norte & $y=1,597+0,039 x$ & 0,064 & 0,203 & - \\
\hline & Extremo Norte & $y=1,602+0,085 x$ & 0,053 & 0,249 & - \\
\hline & Polo Colatina & $y=2,802+0,074 x$ & 0,164 & 0,036 & 4,2 \\
\hline & Noroeste 1 & $y=-0,036+0,180 x$ & 0,457 & 0,001 & 60,7 \\
\hline & Noroeste 2 & $y=1,140+0,194 x$ & 0,482 & 0,001 & 39,1 \\
\hline & Polo Cachoeiro & $y=1,586+0,107 x$ & 0,353 & 0,001 & 72,9 \\
\hline & Caparaó & $y=4,701+0,045 x$ & 0,039 & 0,324 & - \\
\hline
\end{tabular}

* Padronizado pelo método direto; ** não foi possível ajustar um modelo, pois só foi observado 1 óbito em 2005. $y$ : incidência; $x$ : ano.

\section{DISCUSSÃO}

Segundo a OMS, os números de mortes por 100.000 habitantes são influenciados pela distribuição etária da população. Por isso, a padronização das taxas de mortalidade pode ajustar as diferenças na distribuição etária da população ${ }^{15}$. No presente trabalho, as padronizações por faixas etárias pelo método direto (dados geral e por sexo) mostraram-se significativamente diferentes das taxas não padronizadas, sendo por isso adotadas como referência.

De modo mais global, observou-se, nas últimas cinco décadas, uma tendência de aumento das taxas de suicídio, levando-o a constituir-se como uma importante questão de saúde pública em todo o mundo. Merece destaque o fato de que esse aumento ocorreu, principalmente, graças à elevação progressiva das taxas de suicídio entre os homens, ao passo que as taxas entre as mulheres cresceram em ritmo bem inferior ao longo do tempo. Dessa forma, em nível global, enquanto as taxas entre os homens subiram 49\% entre 1950 e 1995, as taxas entre as mulheres subiram 33\% ${ }^{16}$. No presente estudo, também se verificou uma taxa de crescimento entre os homens $(23,8 \%)$, ao passo que, entre as mulheres, a taxa se manteve estável. Meneghel et al. ${ }^{11}$, ao apresentarem os dados epidemiológicos sobre suicídio no Rio Grande do Sul, na série histórica de 1980 a 1999, verificaram que os coeficientes de mortalidade apresentaram tendências ascendentes para o suicídio entre os homens e levemente descendentes entre as mulheres.

Um fato bem documentado acerca da epidemiologia do suicídio é a relação homem/mulher, que, segundo dados da OMS, tem uma taxa global de 3:1, sendo essa relação mantida ao longo do tempo em quase todos os países com dados disponíveis ${ }^{17}$.

Os resultados desta pesquisa também destacam tal relação (3,7:1), apontando maior ocorrência do fenômeno entre os homens, e esses resultados são semelhantes aos encontrados em outros estudos, como os de Marín-León e Barros $^{18}$, que, ao analisarem a tendência da mortalidade por suicídio, entre 1976 e 2001, em município do interior paulis- 
ta, observaram que a razão entre as taxas dos sexos aponta uma mortalidade masculina importante, superior a 2,7 para cada suicídio feminino. Com isso, além de apontarem uma maior mortalidade masculina por suicídio, os autores destacam que os dois períodos com razões de taxas mais elevadas (1988-1991 e 1998-2000) se caracterizaram pela diminuição das taxas femininas abaixo de um suicídio/100 mil mulheres.

Viana et al. ${ }^{19}$, ao descreverem e avaliarem a incidência de suicídio em indivíduos procedentes da Associação dos Municípios da Região de Laguna (Amurel, SC), no período de 2001 a 2005, encontraram maior mortalidade por suicídio na população masculina do que na feminina. Parente et al. ${ }^{20}$, ao caracterizarem casos de suicídio no Município de Teresina (PI), no período de 2000 a 2005, encontraram um predomínio de óbitos por suicídio para o sexo masculino (6 a 11 suicídios por 100 mil/habitantes) em relação ao feminino (1,8 a 4 suicídios por 100 mil/habitantes), com marcante diferença em todos os anos. Também Lovisi et al. ${ }^{21}$, com o objetivo de desenvolver uma análise epidemiológica das taxas de mortalidade por suicídio no Brasil, por regiões e capitais do país, entre 1980 e 2006, destacaram ter encontrado um maior índice de mortalidade entre os homens, com um aumento de $52 \%$ durante o período estudado (de 2,5 a 3,8).

Diversas hipóteses foram levantadas para tentar explicar essa diferença entre os sexos, sendo uma constatação o fato de que os métodos escolhidos pelos homens são, geralmente, mais letais do que os escolhidos pelas mulheres, o que os leva a ter maior possibilidade de suicídio do que elas ${ }^{16,22}$.

A menor ocorrência de suicídio entre as mulheres tem sido atribuída a vários fatores, como a baixa prevalência de dependência do álcool; a maior religiosidade; o envolvimento social e o desempenho de papéis de mãe, esposa e filha, durante a vida. Além disso, as mulheres conseguem reconhecer mais precocemente sinais de riscos para depressão, suicídio e doença mental, além de buscarem mais ajuda em momentos de crise e de participarem mais das redes de apoio social ${ }^{11}$. Quanto aos homens, estes desempenham comportamentos que predispõem ao suicídio (a competitividade, a impulsividade e o maior acesso a tecnologias letais), sendo ainda mais sensíveis às instabilidades econômicas, como desemprego e o empobrecimento etc., fatores esses que podem levar ao suicídio ${ }^{23}$.

Em relação à idade, as taxas de suicídio são cada vez mais elevadas, à medida que se aumenta a faixa etária do grupo estudado ${ }^{16}$. Tal fato foi observado na presente pesquisa, visto que as maiores tendências de crescimento ocorreram nas faixas etárias acima de 30 anos (30 a 39 anos, 16,4\%; 40 a 49 anos, 44,7\% e 50 a 59 anos, 72,3\%).

Lovisi et al. ${ }^{21}$, ao compararem as taxas de mortalidade por suicídio no Brasil entre 1980 e 2006, segundo os grupos de idade, observaram a predominância em indivíduos com mais de 70 anos (uma média de 7,8 mortes por 100.000 habitantes), seguida por pessoas com idade entre 50 e 59 anos e indivíduos com idade entre 60 e 69 anos de idade (uma média de 6,8 e 6,7 mortes por 100.000 habitantes, respectivamente). Quando observadas ao longo do tempo (1980 a 2006), as taxas de suicídio cresceram mais entre os indivíduos com idade entre 20 e 59 anos (30\%) do que entre aqueles com idade maior que 60 anos (19\%). Vansan ${ }^{24}$, analisando dados do Município de Ribeirão Preto, encontrou o equivalente a 40,6\% dos suicídios na faixa etária de 20 a 29 anos; 24,6\% na faixa etária de 30 a 39 anos, e 17,4\% na faixa etária de 40 a 49 anos. Silva et al. ${ }^{25}$, ao estudarem os casos de suicídio registrados no Instituto Médico Legal (IML) da cidade de Salvador de janeiro de 1996 a dezembro de 1997, definiram faixas etárias diferentes, mostrando que a faixa etária de 21 a 30 anos apresentou maior ocorrência de suicídio (24,9\%), seguida pela faixa de 31 a 40 anos (24,5\%) e de 16 a 20 anos (16,6\%).

Destaca-se que dados do Brasil referentes às duas últimas décadas indicam que a população cujas taxas de suicídio mais cresceram corresponde ao estrato dos jovens de 15 a 24 anos $^{26}$. Segundo dados da OMS, em 1998, 55\% dos suicídios foram cometidos por pessoas entre 5 e 44 anos de idade. Em 2004, $76,2 \%$ dos óbitos por suicídio no país concentraram-se na faixa etária de 20 a 59 anos $^{12}$. Esses dados refletem uma tendência mundial de crescimento das taxas de suicídio nos jovens, a ponto de estes passarem a ser o principal grupo de risco em um terço dos países ${ }^{16}$. A presente pesquisa também identificou tendência crescente para a faixa etária de 10 a 14 anos, a qual passou de uma taxa de 0, em 1980, para 1,4 por 100.000 habitantes, no ano de 2006. Lovisi et al. ${ }^{21}$ encontraram resultados semelhantes, ao destacarem que, apesar de mais baixa que a das demais faixas etárias, a taxa de suicídio identificada em sua pesquisa para o grupo com idades entre 10 e 14 anos foi de 20\%. Encontraram também um aumento de 27,6\% das mortes por suicídio entre os indivíduos de 15 a 24 anos. Souza et al. ${ }^{7}$, em estudo realizado em nove capitais brasileiras, destacaram os altos índices de suicídio entre os jovens e chamaram a atenção para o fato de esses brasileiros estarem morrendo, cada vez mais, por causas violentas. Eles apontam a grande pressão ocupacional e o aumento da competitividade no mercado de trabalho como fatores que tornam a população jovem brasileira particularmente vulnerável ao risco de suicídio.

No que se refere à distribuição dos casos de suicídio no Espírito Santo, verifica-se que são poucos os estudos que abordam especificamente a questão do suicídio no Estado. Entre esses estudos, estão os estudos de Tavares ${ }^{27}$, que, com o objetivo de investigar o perfil epidemiológico dos óbitos por causas externas ocorridos no Espírito Santo, na população residente (de 1979 a 2003), verificou a ocorrência de aumento nas taxas de mortalidade por suicídio ao longo dos anos estudados. Em 1979, o risco de morrer por esse motivo no Espírito Santo era de 2/100 mil habitantes, passando, em 2003, para 4,7/100 mil habitantes, ou seja, um incremento de 135\%. Jacobson et al. ${ }^{28}$, ao analisarem a tendência da mortalidade por causas externas no Estado do Espírito Santo em série histórica (de 1994 a 2005), de acordo com grupos específicos (sexo e faixas etárias), verificaram que, para as lesões 
autoprovocadas, a taxa de mortalidade apresentou tendência ascendente significativa durante todo o período. Bastos et al. ${ }^{29}$, com o objetivo de analisar o contexto socioeconômico e espacial da incidência da mortalidade por homicídio, suicídio e acidente de trânsito na capital Vitória, verificaram que, no período de 2000 a 2003, ocorreram 828 óbitos por acidentes e violência, os quais representaram 17\% do total de óbitos do município. Destes, 6\% referiram-se a óbitos por suicídio.

A distribuição dos casos de suicídio no Estado do Espírito Santo segundo suas macro e microrregiões possibilitou a identificação das áreas de risco, fato essencial, segundo a OMS, a qual reforça a necessidade de se buscarem explicações para as variações dos índices de suicídio em seu contexto local. Isso aponta para a necessidade de a vigilância epidemiológica e a pesquisa local serem apropriadas, de forma a favorecer uma melhor compreensão desse grave problema de saúde pública e, assim, melhorar as possibilidades de prevenção ${ }^{17}$.

Nessa perspectiva, ao se analisarem os resultados referentes às tendências de crescimento das taxas de suicídio segundo suas macro e microrregiões, deve-se lembrar de que são vários os fatores de risco para o suicídio, os quais podem ser definidos por meio de estudos epidemiológicos que resgatam as informações vinculadas aos casos de óbitos por suicídio registrados oficialmente, incluindo os métodos escolhidos para o ato. Também é importante a informação sobre os fatores demográficos, fatores psiquiátricos, fatores médicos, fatores relacionados ao comportamento suicida, internação hospitalar e contatos com tratamentos médi$\cos ^{30}$, cabendo, assim, o desenvolvimento de novas pesquisas que aprofundem a questão nas áreas identificadas pela presente pesquisa.

\section{CONCLUSÃO}

As taxas de suicídio diferiram significativamente entre homens e mulheres. Os dados encontrados mostram que a mortalidade por suicídio entre os homens foi mais elevada que a encontrada entre as mulheres, o que coincide com dados da literatura sobre o tema. Quanto à faixa etária, os resultados encontrados apontaram tendência de crescimento nas faixas de 10 a 14 anos; 30 a 39 anos; 40 a 49 anos e 50 a 59 anos. Foram encontradas ainda disparidades quanto à distribuição dos óbitos por suicídio segundo as macro e microrregiões do Estado do Espírito Santo, com tendência de crescimento das taxas nas macrorregiões Metropolitana, Noroeste e Sul, mantendo-se estáveis na macrorregião Norte. Quanto às microrregiões, metade delas apresentou tendência de crescimento.

\section{REFERÊNCIAS}

1. Bertolote JM, Fleishmann A. Suicide and psychiatric diagnosis: a worldwide perspective. World Psychiatry. 2002;1(3):181-5.
2. Bahls SC, Botega NJ. Epidemiologia das tentativas de suicídio e dos suicídios. In: Mello MF, Mello AF, Kohn R, editores. Epidemiologia da saúde mental no Brasil. Porto Alegre: Artmed; 2007. p. 151-71.

3. Organização Mundial da Saúde (OMS). Prevenção do suicídio: manual para médicos clínicos gerais. Genebra: OMS; 2000.

4. World Health Organization (WHO) (1999). Figures and facts about suicide. (Doc. WHO/ MNH/MBD/99.1). Genebra: OMS; 1999.

5. Gawryszewski VP, Koizumi MS, Mello-Jorge MHP. As causas externas no Brasil no ano 2000: comparando a mortalidade e a morbidade. Cad Saúde Pública. 2004;20(4):995-1003.

6. Mello-Santos C, Wang YP, Bertolote JM. Epidemiology of suicide in Brazil (1980-2000): characterization of age and gender rates of suicide. Revista Brasileira de Psiquiatria. 2005;27(2):131-4.

7. Souza ER, Minayo MC, Malaquias JV. Suicídio de jovens nas principais capitais do Brasil. Cad Saúde Pública. 2002;18(3):673-83.

8. Werlang BSG, Botega NJ. Comportamento suicida. Porto Alegre: Artmed; 2004.

9. Vasconcelos AMN. A qualidade das estatísticas de óbitos no Brasil. Rev Bras Estudos Pop Brasília. 1998;15(1):116-24.

10. Baptista MN. Suicídio e depressão. Atualizações. Rio de Janeiro: Guanabara Koogan; 2004.

11. Meneghel SN, Victora CG, Faria NMX, Carvalho LA, Falke JW. Características epidemiológicas do suicídio no Rio Grande do Sul. Rev Saúde Pública. 2004;38(6):804-10.

12. Brasil. Saúde Brasil 2006: uma análise da situação de saúde no Brasil. Brasília: Ministério da Saúde; 2006.

13. Departamento de Informática do Sistema Único de Saúde (DATASUS). População residente. [acesso em 2009 Nov 03]. Disponível em: http://tabnet.datasus.gov.br/cgi/tabcgi. exe?ibge/cnv/popes.def

14. Vermelho LL, Costa AJL, Kale PL. Indicadores de saúde. In: Medronho RA, et al. Epidemiologia. São Paulo: Atheneu; 2006. p. 33-55.

15. World Health Organization (WHO). WHO Statistical Information System (WHOSIS). Age-standardized mortality rates by cause (per 100000 population). [cited 2009 Nov 26]. Available from: http://www.who.int/whosis/indicators/compendium/2008/1mst/en/index.html

16. Volpe FM, Corrêa H, Barrero SP. Epidemiologia do suicídio. In: Corrêa H, Barrero SP. Suicídio: uma morte evitável. São Paulo: Atheneu; 2006. p. 11-27.

17. Organização Mundial da Saúde (OMS). Relatório sobre a saúde no mundo 2001 - saúde mental: nova concepção, nova esperança. Genebra; 2001.

18. Marín-León L, Barros MBA. Mortes por suicídio: diferenças de gênero e nível socioeconômico. Rev Saúde Pública. 2003;37(3):357-63

19. Viana GN, Zenkner FM, Sakae TM, Escobar BT. Prevalência de suicídio no Sul do Brasil, 20012005. J Bras Psiquiatr. 2008;57(1):38-43.

20. Parente ACM, Soares RB, Araújo ARF, Cavalcante IS, Monteiro CFS. Caracterização dos casos de suicídio em uma capital do Nordeste Brasileiro. Rev Bras Enferm Brasília. 2007;60(4):377-81.

21. Lovisi GM, Santos SA, Legay L, Abelha L, Valencia E. Análise epidemiológica do suicídio no Brasil entre 1980 e 2006. Rev Bras Psiquiatr. 2009;31(Supl II):S86-93.

22. Macente LB, Santos EG, Zandonade E. Tentativas de suicídio e suicídio em município de cultura Pomerana no interior do estado do Espírito Santo. J Bras Psiquiatr. 2009;58(4):238-44.

23. Leal OF. Suicídio honra e masculinidade na cultura gaúcha. Cad Antropologia UFRGS. $1992 ;(6): 7-21$.

24. Vansan GA. Aspectos epidemiológicos comparativos entre tentativas de suicídio e suicídios no município de Ribeirão Preto. J Bras Psiquiatr. 1999;48(5):209-15.

25. Silva JA, Silva CN, Silva Jr, Silva LN, Silva DN. Epidemiologia do suicídio na cidade de Salvador (BA). Rev Bras Neurologia Psiquiátrica. 1999;3(1):19-25.

26. Wang YP, Mello-Santos C, Bertolote JM. Epidemiologia do suicídio. In: Meleiro A, Teng CT, Wang YP. Suicídio: estudos fundamentais. São Paulo: Segmento Farma; 2004. p. 97-108.

27. Tavares FL. A mortalidade por causas externas no Espírito Santo, de 1979 a 2003. [Dissertação]. Vitória: Universidade Federal do Espírito Santo. Programa de Pós-graduação em Saúde Coletiva, 2005

28. Jacobson LSV, Andrade CLT, Carmo CN, Mourão DS, Hacon SS. Trend in mortality due to external causes in the State of Espírito Santo, Brazil, from 1994 to 2005. Rev Bras Epidemiol [online]. 2009;12(1):82-91.

29. Bastos MJRP, Pereira JA, Smarzaro DC, Costa EF, Bossanel RCL, Oliosa DMS, et al. Análise ecológica dos acidentes e da violência letal em Vitória, ES. Rev Saúde Pública. 2009;43(1):123-32.

30. Meleiro AMAS, Teng CT. Fatores de risco de suicídio. In: Meleiro A, Teng CT, Wang YP. Suicídio: estudos fundamentais. São Paulo: Segmento Farma. 2004; p. 109-31. 